\title{
Use of hospital beds: a cohort study of admissions to a provincial teaching hospital
}

\author{
Peter Anderson, Jill Meara, Sally Brodhurst, Sara Attwood, Mary Timbrell, Alex Gatherer
}

\begin{abstract}
An instrument was developed to study the use of hospital beds and discharge arrangements of a cohort of 847 admissions to the John Radcliffe Hospital, Oxford, for a three week period during February-March 1986 . For only $38 \%$ of bed days were patients considered to have medical, nursing, or life support reasons for requiring a provincial teaching hospital bed. The requirements for a bed in the hospital decreased with the patient's age and length of stay in hospital. For only a tenth of patients was the general practitioner concerned in discussions with hospital staff about the patient's discharge and less than one third of patients had been given more than 24 hours' notice of discharge.

Several features might increase the proportion of bed days that are occupied by patients with positive reasons for being in hospital. Among these are an increased frequency of ward rounds by consultants, or delegating discharge decisions by consultants to other staff; providing diagnostic related protocols for planning the length of stay in hospital; planned discharges; and providing liaison nurses to help with communication with primary care staff.
\end{abstract}

\section{Introduction}

Hospitals throughout the world face the same problem of balancing quality of care against cost containment. In the United States cost containment in hospitals is greatly influenced by medical insurance companies which determine through payment the appropriateness of hospital admissions and length of stays. In the United Kingdom general practitioners have a controlling influence on admissions to hospitals. Length of stay is often determined how many people are waiting for a bed. Several authors have analysed the appropriateness of hospital admissions and length of been undertaken in the United Kingdom. ${ }^{6-8}$

We carried out a study on the use of beds by patients admitted to the John Radcliffe Hospital, Oxford, for three weeks during February-March 1986. We developed and used an instrument aimed at measuring the use of beds by an interview with nursing and medical staff. The instrument is similar to the Boston appropriateness evaluation protocol, ${ }^{4}$ which measures unnecessary days of hospital care by audit of medical records.

\section{Department of Commu \\ Medicine, Oxfordshire \\ Health Authority, \\ Headington, Oxford \\ OX3 9DZ}

Peter Anderson, MRCGP, senior registrar in community medicine

Jill Meara, MSc, senior registrar in community medicine

Sally Brodhurst, cosw, research social worker

Sara Attwood, management trainee

Mary Timbrell,

administrative assistant

Alex Gatherer, FFCM, district medical officer

Correspondence to: $\mathrm{Dr}$ Anderson.

\section{Methods}

The hospital-The John Radcliffe Hospital is a teaching hospital that acts as the main acute district general hospital for the city of Oxford and surrounding towns and villages. Acute stay beds for patients with respiratory, gynaecological, and neurological problems are available at other hospitals in the city. The hospital has one of the highest throughputs of patients for any acute unit in England and Wales.

The cohort-All admissions to the hospital between 24 February and 16 March 1986 under medical, geriatric, and general surgical consultant physicians were included. The cohort excluded obstetric and gynaecological patients and children under age 16. Day patients were also excluded. The patients were followed up prospectively until discharge or death. stay in the United States, ${ }^{1-5}$ but few such studies have

Data collection-The following data were collected for all patients admitted: name, sex, date of birth, date of admission, type of admission (emergency, elective), specialty, diagnosis, and date of discharge or death. Information was collected on which wards the patients stayed, and whether the wards were the usual wards for that particular consultant's patients. The number of movements between wards for each patient was recorded for each day in hospital. Whether or not the patient was on the "wrong" ward was determined, wrong ward being defined as not the usual ward used by the patient's consultant. The short stay admissions wards and intensive therapy and coronary care units were not defined as wrong wards. The days of ward rounds and days of "on take" for emergency admissions for each consultant were recorded.

Patient interviews-One in 10 patients in the study were interviewed while on the wards to discover their views about their hospital stay and discharge plans. Nine tenths of the patients interviewed in hospital were seen again at home between 10 and 18 days after discharge.

Questionnaire survey of general practitioners-For all patients discharged the general practitioners were asked by questionnaire when and how they were informed of the patients' discharges.

Bed study instrument-For every alternative patient who was in hospital the day after his or her admission, data were collected on use of hospital beds using the bed study instrument. This was administered by two community physicians ( $\mathrm{P} \mathrm{A}$ and $\mathrm{J} \mathrm{M}$ ) at interview with the senior nurse on each ward. The bed study instrument consists of two parts. Part 1 (table I)

TABLE I-The bed study instrument criteria for appropriateneness of hospital days

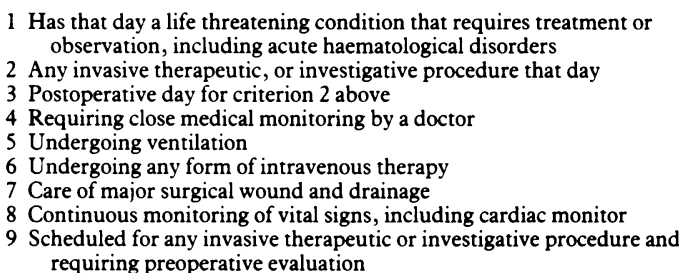

TABLE II -Reasons for not being at home

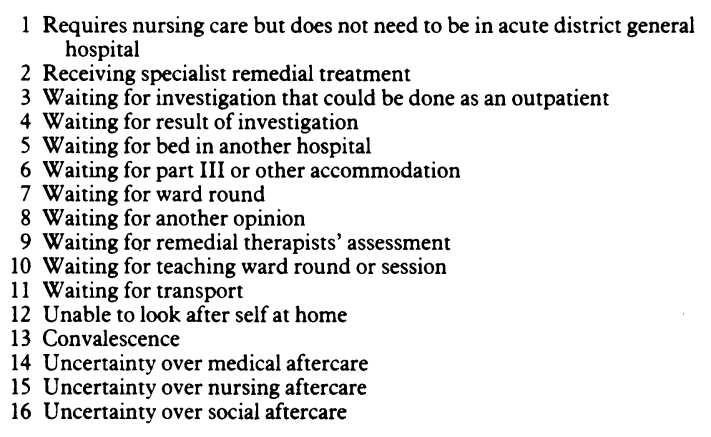

consists of nine criteria related to medical, nursing, and life support services. The patient was considered to have a positive reason to be in hospital if any criterion was met. Part 2 (table II) consists of 16 criteria to determine why the patient was not at home if none of 
the nine criteria of part 1 applied. The criteria of part 2 are listed in a hierarchical order - that is, when more than one criterion was given the highest criterion in the order was accepted.

Validity of instrument-For a $6 \%$ random sample of bed days the interview with the senior nurse using the bed study instrument was validated by interview with the appropriate senior registrar of the patient. For $88 \%$ of the cases senior registrars and the nurses were in agreement on whether or not there was a positive reason for use of the hospital bed (Kendall's coefficient of concordance, $\left.{ }^{9} \mathrm{~W}=0 \cdot 88, \mathrm{p}<0 \cdot 00001\right)$. This level of agreement is better than that achieved between physicians assessing the appropriateness of bed days by audit of medical records.

\section{Results}

During the three weeks of the study there were 847 admissions: $584(69 \%)$ were for emergencies and 263 (31\%) for elective procedures; $432(51 \%)$ patients were admitted under the care of a physician, $322(38 \%)$ under a surgeon, and $93(11 \%)$ under a geriatrician. Thirty six consultants admitted patients, with an average of 23 patients per consultant.

The mean length of stay was $7 \cdot 7$ days for emergency admissions and 5.0 days for elective admissions. The mean length of stay for medical patients was 6.0 days, surgical patients 6.2 days, and geriatric patients 13.4 days.

The patients who were admitted as emergencies accounted for $77 \%$ of bed days; medical patients accounted for $44 \%$ of bed days, surgical patients for $34 \%$, and geriatric patients for $22 \%$. Patients over age 65 accounted for just under half $(398 ; 47 \%)$ of admissions and $65 \%$ of bed days. Patients over 85 accounted for less than a tenth $(59 ; 7 \%)$ of admissions and $13 \%$ of bed days.

Less than a third $(254 ; 30 \%)$ of the patients were moved at least once between wards during their hospital stay, and half $(415 ; 49 \%)$ spent one or more days on the wrong ward during their hospital stay.

\section{BED STUDY INSTRUMENT}

Four hundred and five patients were eligible for assessment with the bed study instrument. The instrument was completed for $328(81 \%)$ of these patients but not for the remaining 77 because either they had been discharged or they had died before the first ward visit. Table III gives the distribution of use of hospital beds.

TABLE III -Distribution of bed day use by type of admission

\begin{tabular}{|c|c|c|c|}
\hline & \multicolumn{2}{|c|}{ Type of admission } & \multirow[b]{2}{*}{$\begin{array}{c}\text { Total } \\
(n=2317)\end{array}$} \\
\hline & $\begin{array}{c}\text { Emergency } \\
(\mathrm{n}=1828) \\
(\%)\end{array}$ & $\begin{array}{c}\text { Elective } \\
(\mathrm{n}=489) \\
(\%)\end{array}$ & \\
\hline \multicolumn{4}{|l|}{ Positive reasons for being in hospital: } \\
\hline Life threatening condition $(1)^{\star}$ & $3 \cdot 3$ & 1.6 & $3 \cdot 0$ \\
\hline Undergoing invasive procedure (2) & $3 \cdot 0$ & $16 \cdot 8$ & 5.9 \\
\hline Requiring specialist skills $(3-8)$ & $24 \cdot 7$ & $29 \cdot 2$ & $25 \cdot 6$ \\
\hline Scheduled for invasive procedure (9) & $3 \cdot 0$ & $6 \cdot 3$ & $3 \cdot 7$ \\
\hline \multicolumn{4}{|l|}{ Reasons for not being at home: } \\
\hline \multicolumn{4}{|l|}{ Waiting, social reasons $(6,11,12,13$, } \\
\hline \multirow{3}{*}{$\begin{array}{l}\text { Requiring nursing care }(1,2,5,9,15) \\
\text { Waiting, medical reasons }(3,4,7,8, \\
10,14)\end{array}$} & $38 \cdot 0$ & $\begin{array}{r}4.5 \\
32.5\end{array}$ & $\begin{array}{l}10.5 \\
36.9\end{array}$ \\
\hline & & & \\
\hline & $15 \cdot 9$ & $9 \cdot 2$ & $14 \cdot 5$ \\
\hline
\end{tabular}

*Numbers in parentheses refer to numbers in table I.

tNumbers in parentheses refer to numbers in table II.

Overall, the patients sampled were considered to have positive reasons for being in this hospital on $38 \%$ of the total bed days as judged by the instrument. The proportion of bed days with positive reasons was as follows: $52 \%$ in surgical beds, $37 \%$ in medical beds, and $22 \%$ in geriatric beds; $34 \%$ of bed days for emergency admissions were judged to have positive reasons compared with $48 \%$ of bed days for elective admissions. The proportion of patient bed days with positive reasons was related to the patient's age, being $50 \%$ for patients aged $16-64$ years but only $19 \%$ for patients over 84 .

The proportion of bed days with positive reasons decreased steadily from $74 \%$ on the first day after admission to $22 \%$ on the eighth day after admission. Thereafter, the proportion remained unchanged for increasing lengths of stay. It increased from $35 \%$ for patients who had one move during their hospital stay to $94 \%$ for patients who had four moves. It decreased from $43 \%$ for patients who were never on the wrong ward to $30 \%$ for those who spent all of their time on the wrong ward.

The proportion of bed days with positive reasons was related to days of consultant ward rounds and days when consultants were "on take" for emergency admissions. For the three days leading up to a ward round the proportion remained constant at $33 \%$. On the day after a ward round the proportion increased to $62 \%$ and then decreased to $40 \%$ by the third day after the ward round. For the three days leading up to an "on take" day the proportion remained constant at $40 \%$, increasing to $60 \%$ the day after and reducing to $40 \%$ by the third day after the "on take" day.

Patient interviews-Discharge plans had been discussed with three fifths of patients during their hospital stay. Discussion of discharge plans was not related to the patient's length of stay. When interviewed at home nine tenths of patients said that they were satisfied with the timing of their discharge, though less than a third of all patients interviewed had been given more than 24 hours' notice of discharge.

General practitioner's discharge questionnaire -548 of $610(90 \%)$ questionnaires sent to the general practitioners of patients discharged were completed. For only one quarter of patients was the general practitioner informed of the discharge on or before the day it had occurred. For only a tenth of patients did the general practitioner discuss the patient's discharge with hospital staff. General practitioners were not more concerned in the discharge plans of patients with more complex social problems or of very elderly patients.

\section{Discussion}

We developed the bed study instrument, which can be used at interview with nursing or medical staff to determine bed use. The validity of the instrument can be judged according to how well it replicates the assessment of the experienced nursing and medical staff. The high degree of concordance between doctors and nurses suggests that the instrument is reliable. We adopted the instrument to be used at interview rather than by an audit of medical records because we believed that it would provide more accurate and complete information concerning the patient's hospital stay. Using the instrument, for only $38 \%$ of bed days during the three week period during February to March 1986 was the patient considered to have positive reasons for being in hospital.

How does this result compare with other studies? In a study at the Radcliffe Infirmary, Oxford, during March 1970 it was estimated that $32 \%$ of bed days could have been saved by using alternatives to admission and by earlier discharge ${ }^{8}$ In a study examining 1132 hospital admissions to over 100 hospitals in the United States during 1974-82 it was found that $60 \%$ of hospital bed days were "appropriate."s Appropriateness was measured by auditing medical records using a protocol similar to the bed study instrument. The 
difference between the two studies may be due to different case mixes. The John Radcliffe Hospital has a low proportion of elective admissions. Such admissions were found to have a higher proportion of bed days judged to have positive reasons.

There are several possibilities for increasing the proportion of bed days that are occupied by patients with positive reasons for being in hospital. Since ward rounds by consultant physicians seem to be major determinants of the date of a patient's discharge increasing the frequency of ward rounds at which discharge decisions are made, delegating responsibility for discharge decisions to other staff, and providing diagnostic related protocols to plan patients' length of stay in hospital would all promote earlier discharge. In North America length of stay is partly determined by payment for agreed length of stay for diagnostic groups. Using the Delphi technique ${ }^{1011}$ it is possible to reach agreement on suitable lengths of stay for diagnostic groups. In a North American study conducted in four general hospitals feedback of information to physicians about appropriate use of beds reduced inappropriate bed use by between two thirds and two and a half total days compared to the inappropriate bed use for patients of physicians who got no feedback. ${ }^{12}$

In several previous studies the findings suggested that planned discharge allows more effective use of beds, ${ }^{13-16}$ but there was little evidence of discharge planning in our study. We are now auditing the case notes for evidence of discharge planning and auditing general practitioner referral letters for evidence of information relevant to a patient's discharge.

Providing liaison nurses to hospital wards might help achieve earlier planned discharge of patients. The task of the nurse would not be to take over the role of the ward staff in dealing with discharge but to facilitate this role. This could be achieved by the nurse inquiring about discharge plans for patients on the ward, providing information about primary care and social services, helping with communication with primary care staff, ensuring that discharge notes are sent to the general practitioner on time, and troubleshooting particularly complex or difficult problems. Such a role could be evaluated by a randomised controlled trial assessing the impact of the liaison nurse on discharge planning and length of hospital stay.

We thank the John Radcliffe Unit Management Executive for their consent and help in executing this study; Lynda Meer and her colleagues for providing the admissions data; Mary Stokes and Tracey Haren for providing office space; Fiona Rennie and Janet Robertson for help in preparing the data; and all the nurses, senior registrars, consultants, general practitioners, and patients for their cooperation and interest in providing data for the study.

1 Borchardt PJ. Non acute profiles: evaluation of physicians' non acute utilization of hospital resources. $Q R B$ 1981;7 (Nov):21-6.

Restuccia JD, Gertman PM, Davno SJ, Kreger BE, Llenhart GM. A comparative analysis of appropriateness of hospital use. Health Aff 1984;3: comparat $130-8$.

3 Zimmer JG. Length of stay and hospital bed misutilization. Med Care 1974;12:453-62.

4 Gertman PM, Restuccia JD. The appropriateness evaluation protocol: a technique for assessing unnecessary days of hospital care. Med Care 1981:19:855-71.

5 Siu AL, Sonnenberg FA, Manning WG, et al. Inappropriate use of hospitals in a randomized trial of health insurance plans. N Englf F Med 1986;315:1259-66. 6 Torrance N, Lawson JAR, Hogg B, Knox JDG. Acute admissions to medical beds. I R Coll Gen Pract 1972;22:211-9.

7 Lagoe RJ. Differences in hospital discharge rates. A community-based analysis. Med Care 1986;24:868-72.

8 Loudon ISL. The demand for hospital care. Oxford: United Oxford Hospitals Trust, 1970.

9 Siegel S. Nonparametic statistics for the behavioural sciences. London, McGrawHill, 1946.

10 Rosser RM. Reliability and application of clinical judgement in evaluating the use of hospital beds. Med Care 1976;14:39-47.

11 Holloway DC, Holton JP, Goldberg GA, Restuccia JD. Development of hospital levels of care criteria. Health Care Management 1976;1:61-72

12 Restuccia JD. The effect of concurrent feedback in reducing inappropriate hospital utilization. Med Care 1982;20:46-62.

13 Cable EP. Mavers SP. Discharge planning effect on length of hospital stav. Arch Phys. Med Rehabil 1983;64:75-60.

14 Schroager J, Halman M, Myers D, Nichols R, Rosenblum L. Impediments to the course and effectiveness of discharge planning. Soc Work Health Care 1978;4:65-79

15 Cunningham LS. Early assessment for discharge planning. QRB 1981;7(Oct): $11-6$

16 Jessee WF, Doyle BJ. Discharge planning: using audit to identify areas that need improvement. $Q R B$ 1979;5:25-8,

Accepted 19 fuly 1988

\section{ANY QUESTIONS}

Are febrile convulsions in a child a contraindication to combined measles, mumps, and rubella vaccination and to measles and pertussis immunisation?

Convulsions are the commonest severe sequel of measles (estimates range from one per 200 to 500 cases). They are commoner when the child has had febrile convulsions; since measles is endemic in Britain such a history emphasises the importance of children being immunised against measles, mumps, and rubella. The risk is much smaller (one eighth to one tenth the magnitude) that a convulsion will be precipitated by the febrile reaction commonly occurring five to 10 days after immunisation.'

Before 1988 the Department of Health's advice was that a personal history of convulsions was a contraindication to pertussis immunisation. It is now realised, however, that this leaves such children at a greater risk from the disease than from any complication of the immunisation. ${ }^{2}$ The new edition of the DHSS's guidelines makes a personal history of convulsions only a special consideration, ${ }^{3}$ and it is my practice to recommend vaccination for children with febrile convulsions. Most pertussis immunisation should be carried out before the ages when febrile convulsions become common (after 9 months). In young infants afebrile convulsions, associated with underlying neurological conditions such as epilepsy, are commoner. These children are usually also advised to have pertussis immunisation. ${ }^{3}$ With a baby it may be prudent initially to immunise it against diphtheria and tetanus, later catching up with pertussis immunisation when the diagnosis is clear. Otherwise management may be complicated through a deteriorating neurological condition being (wrongly) blamed on the immunisation.
The parents of a child with a tendency to convulsions should be counselled on the management of any fever developing after immunisation. These convulsions usually occur five to 10 days after measles or measles, mumps, and rubella immunisation whereas they take place in the first 72 hours after pertussis immunisation. The advice is the same as when fever is caused by an infection, and doctors vary in their favoured techniques: paracetamol, extra fluids, thin clothing, a cool room, and tepid sponging are all popular procedures. In the United States the recommendation is to give an antipyretic drug routinely for the 72 hours in high risk children. Where the tendency is severe a short course of an anticonvulsant drug is sometimes helpful, an alternative being for parents to have rectal diazepam to hand. In the past an injection of immunoglobulin was sometimes given with measles to prevent its febrile effects. This practice is now contraindicated as it may interfere with the effectiveness of the mumps and rubella components of the combined measles, mumps, and rubella vaccine, which is now being introduced for routine use in Britain. ${ }^{4}-$ ANGUS NICOLL, lecturer, department of tropical medicine, London School of Hygiene and Tropical Medicine, London

1 Measles Vaccine Committee. A report to the Medical Research Council by the measles vaccine committee. BrMed f 1966;i:441-6.

Prensky AL. History of convulsions and use of pertussis vaccine. 7 Paediatrics 1983;107: 244-5.

3 Department of Health and Social Security. Immunisation against infectious disease. London: HMSO, 1988.

4 Department of Health and Social Security. Update of memorandum "immunisation against infectious disease" and introduction of measles, mumps and rubella vaccine. London: DHSS, 1988. (PL/CMO $(88) 19$. 